\title{
Time-of-flight magnetic resonance angiography imaging of a residual arteriovenous malformation nidus after Onyx embolization for stereotactic radiosurgery planning
}

\author{
Technical note
}

\author{
David N. Loy, M.D., Ph.D., ${ }^{1}$ Keith M. Rich, M.D., ${ }^{2}$ Joseph Simpson, M.D., Ph.D., ${ }^{3}$ \\ Ian Dorward, M.D., ${ }^{2}$ Lakshmi Santanam, Ph.D., ${ }^{3}$ and Colin P. Derdeyn, M.D. ${ }^{1,2,4}$ \\ ${ }^{1}$ Mallinckrodt Institute of Radiology and Departments of ${ }^{2}$ Neurosurgery, ${ }^{3}$ Radiation Oncology, and \\ ${ }^{4}$ Neurology, Washington University School of Medicine, Saint Louis, Missouri
}

\begin{abstract}
This report demonstrates that time-of-flight (TOF) MR angiography is a useful adjunct for planning stereotactic radiosurgery (SRS) of large arteriovenous malformations (AVMs) after staged embolization with Onyx.

Onyx (ethylene vinyl copolymer), a recently approved liquid embolic agent, has been increasingly used to exclude portions of large AVMs from the parent circulation prior to SRS. Limiting SRS to regions of persistent arteriovenous shunting and excluding regions eliminated by embolization may reduce unnecessary radiation doses to eloquent brain structures. However, SRS dosimetry planning presents unique challenges after Onyx embolization because it creates extensive artifacts on CT scans, and it cannot be delineated from untreated nidus on standard MR sequences.

During the radiosurgery procedure, MR images were obtained using a GE Signa 1.5-T unit. Standard axial T2 fast spin echo high-resolution images (TR $3000 \mathrm{msec}$, TE $108 \mathrm{msec}$, slice thickness $2.5 \mathrm{~mm}$ ) were generated for optimal visualization of brain tissue and AVM flow voids. The 3D TOF MR angiography images of the circle of Willis and vertebral arteries were subsequently obtained to visualize AVM regions embolized with Onyx (TR $37 \mathrm{msec}$, TE $6.9 \mathrm{msec}$, flip angle $20^{\circ}$ ).

Adjunct TOF MR angiography images demonstrated excellent contrast between nidus embolized with Onyx and regions of persistent arteriovenous shunting within a large AVM prior to SRS. Additional information derived from these sequences resulted in substantial adjustments to the treatment plan and an overall reduction in the treated tissue volume. (DOI: 10.3171/2009.1.FOCUS08246)
\end{abstract}

\begin{abstract}
KEY WORDS • stereotactic radiosurgery • arteriovenous malformation • Onyx • ethylene vinyl copolymer • time-of-flight magnetic resonance angiography $\quad \bullet \quad$ computed tomography
\end{abstract}

$\mathrm{C}$ OMBINATIONS of endovascular embolization and SRS may be used as a treatment strategy for patients with large inoperable intracranial AVMs. Onyx (ethylene vinyl copolymer), a recently approved liquid embolic agent, has been increasingly used in the endovascular treatment of AVMs. ${ }^{7,8}$ The advantages and safety profile of Onyx have been reported previously. ${ }^{3}$ Limiting SRS to regions of persistent arteriovenous shunting and excluding regions eliminated by embolization may reduce unnecessary radiation doses to eloquent

\footnotetext{
Abbreviations used in this paper: AVM = arteriovenous malformation; FOV = field of view; SRS = stereotactic radiosurgery; TOF = time-of-flight.
}

brain structures. During SRS, AVM nidus targeting is performed with CT, MR, and/or catheter angiographic images. Imaging techniques for SRS treatment planning after Onyx AVM embolizations present difficulties that have not been reported. We recently performed SRS in a patient with a large complex AVM after partial embolization with Onyx. Arteriovenous malformations respond to SRS in regions of significant flow defined by TOF MR angiography. ${ }^{4}$ We supplemented our typical imaging during SRS treatment planning with TOF MR angiography that demonstrated contrast between Onyx and regions of persistent arteriovenous shunting. The TOF MR angiography imaging was key in allowing improved identification of residual nidus, which allowed for more accurate dosimetry planning during SRS. 


\section{N. Loy et al.}

Methods
Illustrative Case
This 25-year-old woman had a Spetzler-Martin Grade VI AVM involving the cerebellum, pons, and midbrain. She had presented with hydrocephalus requiring ventriculoperitoneal shunting $\sim 10$ years ago. She has had long-standing severe problems with truncal ataxia, bilateral dysmetria, diplopia, and dysarthria. Over the last 3 years her symptoms have progressed, resulting in requirements for assistance in nearly all activities of daily living. Given her young age, poor prognosis, and progressive severe disabilities, she underwent staged embolization followed by SRS. She underwent a series of staged embolizations with $N$-butyl cyanoacrylate, and most recently with Onyx. Her dysarthria and ataxia improved after endovascular therapy, but again showed progression prior to SRS.

During the radiosurgery procedure, MR images were obtained using a 1.5-T unit (Signa, General Electric Healthcare), which had a gradient strength of $40 \mathrm{mT} / \mathrm{m}$ and a slew rate of $200 \mathrm{~T} / \mathrm{sec} / \mathrm{m}$, by using a phased array linear surface coil. Standard axial T2-weighted fast spin echo high-resolution images (TR 3000 msec, TE 108 msec, slice thickness $2.5 \mathrm{~mm}$ ) were generated for optimal visualization of brain tissue and AVM flow voids. Subsequently, 3D TOF MR angiograms of the circle of Willis and vertebral arteries were obtained to visualize AVM regions embolized with Onyx (TR $37 \mathrm{msec}$, TE $6.9 \mathrm{msec}$, flip angle $20^{\circ}$, FOV 220, phase FOV 0.75 , matrix $200 \times 512$ $\mathrm{mm}$, number of slabs 3 , slices/slab 32, overlap $6 \mathrm{~mm}$, slice thickness $1.4 \mathrm{~mm}$, number of averages 1,1 venous saturation pulse). Contiguous, nonoverlapping spiral CT images were obtained on a Siemens EMOTION SOMATOM. All images were transferred to Leksell Gamma Plan for Gamma Knife planning (Elekta Instrument AB).

\section{Discussion}

The radiopaque component of Onyx, tantalum, produces extensive beam hardening artifacts on CT angiograms, rendering this imaging modality ineffective for postembolization SRS planning in most $\operatorname{cases}^{5}$ (Fig. 1). The T2-weighted MR images are frequently used for SRS planning because these sequences provide maximum contrast between brain tissue and AVM flow voids. However, Onyx also appears hypointense on T2-weighted MR sequences, thus obscuring definition of the flow voids in the partially embolized AVM nidus. ${ }^{5}$

To overcome these obstacles, we used TOF MR angiography as an adjunct to standard T2-weighted imaging for SRS planning in this patient. To our knowledge, this is the first report in which TOF MR angiography has been used for Gamma Knife treatment planning after intracranial AVM embolization with Onyx.

Time-of-flight is an MR imaging technique that uses multiple preparatory radiofrequency pulses to saturate proton spins and suppress the MR signal in stationary tissues. Inflowing blood not present initially in the saturated tissue exhibits an MR signal inside the slab, thus providing vascular contrast without intravenous $\mathrm{Gd}^{2}$

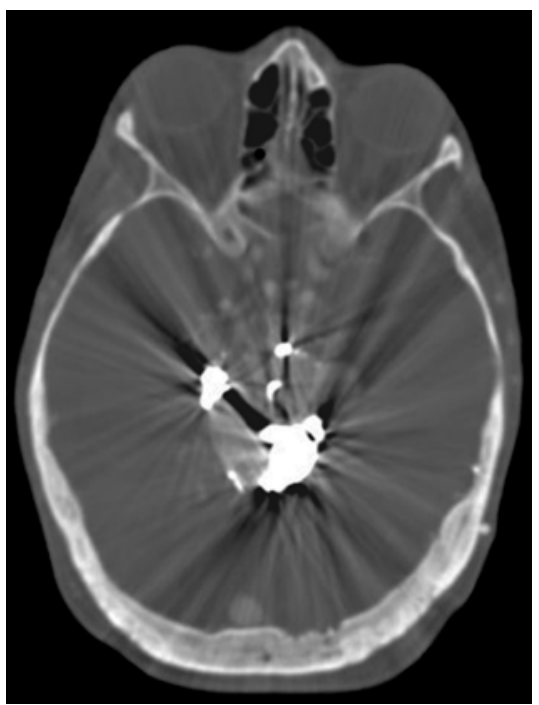

FIG. 1. Transaxial contrast enhanced CT image demonstrating extensive beam hardening artifact after Onyx embolization.

Onyx appears hypointense on TOF MR angiography images, primarily because of its low proton density and the nonferromagnetic properties of tantalum (Fig. 2). Contrast is the result of shunted hyperintense blood flowing into the saturated slab while Onyx, stationary within the AVM and proton-poor, appears as a dark signal void. In this report, adjunct TOF MR angiography images demonstrate excellent contrast between nidus embolized with Onyx and regions of persistent arteriovenous shunting within an AVM prior to stereotactic radiosurgery (Fig. $2 \mathrm{~A}$ and $\mathrm{B})$. Additional information derived from these sequences resulted in substantial adjustments to our treatment plan and an overall reduction in the treated tissue volume (Fig. 2C).

The TOF MR angiography modality is susceptible to signal loss and artifacts primarily related to the length of time required for inflowing blood to pass through a selected 3D image volume. Short repetition times used to maintain proton spin saturation within the stationary image volume may result in significant signal loss within a complex or partially embolized AVM. Additional radiofrequency pulses may be applied before pathologically slowed or turbulent inflowing blood can escape the AVM. This results in intravascular contrast degradation, causing some of the diseased vessels to appear gray, or even isointense to adjacent brain (Fig. 2B). Signal loss within slow flow regions did not obscure AVM nidus embolized with Onyx. However, such effects could be minimized by increasing the repetition time, decreasing the flip angle, or by using higher field strengths to increase signal-tonoise ratios. It should also be noted that methemoglobin within the thrombus can also appear hyperintense because of its short $\mathrm{T} 1$, effectively mimicking persistent flow. ${ }^{6}$ Therefore, careful comparison with high-resolution T2-weighted anatomical sequences is essential for accurate interpretation of TOF MR angiography prior to SRS in our experience.

The TOF MR angiography modality is routinely used 


\section{Time-of-flight MR angiography of AVM nidus after embolization}

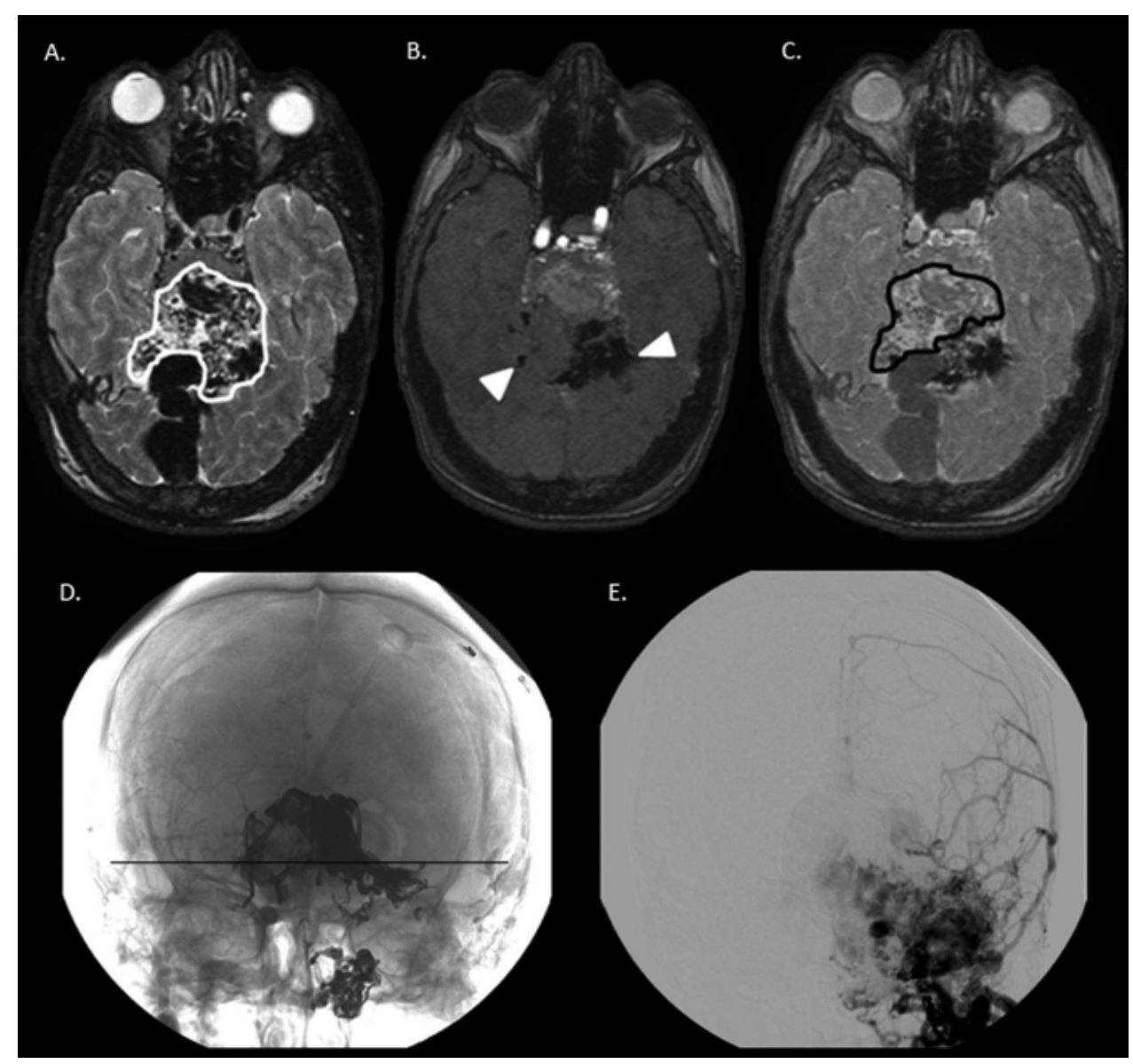

FIG. 2. Representative T2-weighted (A) and TOF (B) MR angiography images of a Spetzler-Martin Grade VI AVM centered in the cerebellum and exerting mass effect on the midbrain. Standard T2-weighted images define brain anatomy and AVM extent (dark flow voids), but do not delineate embolized AVM regions. The TOF MR angiography image (B) provides excellent contrast between Onyx (dark signal voids, arrowheads) and persistent shunts (hyperintense and isointense vessels; compare panels A and $\mathrm{B}$ ). A fusion image $(\mathrm{C})$ combining data from high-resolution T2-weighted and TOF MR angiography source images demonstrates the relationship between dark embolized nidus and regions of persistent shunting. The black outline in panel $\mathrm{C}$ represents a smaller, more accurate treatment region after identification of Onyx-embolized nidus with TOF MR angiography images. Native radiograph $(D)$ and digital subtraction angiography image (E) obtained after left common carotid artery injection, demonstrating the extent and irregular configuration of embolized (D) and residual (E) AVM nidus. The horizontal black line in panel D represents the approximate level of the MR images at the level of the superior cerebellar artery; this was not at the level of the orbits because of differences in image plane angulation between MR and digital subtraction angiography (Townes view) images.

clinically in neuroradiology practices to image the intracranial vasculature. Signal voids have been described within cerebral aneurysms after Onyx embolization. ${ }^{5}$ Despite a report by Bednarz et al. ${ }^{1}$ also describing important planning modifications based on TOF MR angiography prior to AVM stereotactic radiosurgery, this sequence remains underutilized in standard Gamma Knife planning protocols.

\section{Conclusions}

Axial TOF-MR angiography source images provided excellent contrast between Onyx and regions of persistent shunting within a large, incompletely treated AVM. These techniques in combination with standard T2-weighted images are useful for planning staged combination endovascular embolization and SRS.

\section{Disclaimer}

The authors report no conflict of interest concerning the materials or methods used in this study or the findings specified in this paper.

\section{References}

1. Bednarz G, Downes B, Werner-Wasik M, Rosenwasser RH: Combining stereotactic angiography and 3D time-of-flight magnetic resonance angiography in treatment planning for arteriovenous malformation radiosurgery. Int J Radiat Oncol Biol Phys 46:1149-1154, 2000

2. Bitar R, Leung G, Perng R, Tadros S, Moody AR, Sarrazin $\mathrm{J}$, et al: MR pulse sequences: what every radiologist wants to know but is afraid to ask. Radiographics 26:513-537, 2006

3. Cronqvist M, Wirestam R, Ramgren B, Brandt L, Romner B, Nilsson $\mathrm{O}$, et al: Endovascular treatment of intracerebral arteriovenous malformations: procedural safety, complications, and results evaluated by MR imaging, including diffusion and 
perfusion imaging. AJNR Am J Neuroradiol 27:162-176, 2006

4. Petereit D, Mehta M, Turski P, Levin A, Strother C, Mistretta $\mathrm{C}$, et al: Treatment of arteriovenous malformations with stereotactic radiosurgery employing both magnetic resonance angiography and standard angiography as a database. Int J Radiat Oncol Biol Phys 25:309-313, 1993

5. Saatci I, Cekirge HS, Ciceri EF, Mawad ME, Pamuk AG, Besim A: CT and MR imaging findings and their implications in the follow-up of patients with intracranial aneurysms treated with endosaccular occlusion with onyx. AJNR Am J Neuroradiol 24:567-578, 2003

6. Tkach JA, Ruggieri PM, Ross JS, Modic MT, Dillinger JJ, Masaryk TJ: Pulse sequence strategies for vascular contrast in time-of-flight carotid MR angiography. J Magn Reson Imaging 3:811-820, 1993
7. Weber W, Kis B, Siekmann R, Jans P, Laumer R, Kühne D: Preoperative embolization of intracranial arteriovenous malformations with Onyx. Neurosurgery 61:244-252, 2007

8. Weber W, Kis B, Siekmann R, Kuehne D: Endovascular treatment of intracranial arteriovenous malformations with onyx: technical aspects. AJNR Am J Neuroradiol 28:371-377, 2007

Manuscript submitted October 15, 2008.

Accepted January 20, 2009.

Address correspondence to: Colin P. Derdeyn, M.D., Washington University in Saint Louis, 510 South Kingshighway Boulevard, Saint Louis, Missouri 63110. email: derdeync@wustl.edu. 